\title{
Preoperative Laboratory Testing in Patients Undergoing Elective Surgery: An Analysis of Practice at Komofo Anokye Teaching Hospital
}

\author{
Akwasi Antwi-Kusi ${ }^{1, ~ *, ~ B r i g h t ~ I g h o d a r o ~ O b a s u y i ~}{ }^{2}$, William Addison ${ }^{1}$ \\ ${ }^{1}$ Department of Anaesthesia and Intensive Care, Komfo Anokye Teaching Hospital, Kumasi, Ghana \\ ${ }^{2}$ Department of Anaesthesiology, University of Port Harcourt Teaching Hospital, Port Harcourt, Nigeria
}

Email address:

antwikusi@yahoo.com (A. Antwi-Kusi)

*Corresponding author

\section{To cite this article:}

Akwasi Antwi-Kusi, Bright Ighodaro Obasuyi, William Addison. Preoperative Laboratory Testing in Patients Undergoing Elective Surgery: An Analysis of Practice at Komofo Anokye Teaching Hospital. Journal of Anesthesiology. Vol. 5, No. 2, 2017, pp. 5-10. doi: 10.11648/j.ja.20170502.11

Received: February 24, 2017; Accepted: March 14, 2017; Published: March 29, 2017

\begin{abstract}
Background: Perioperative laboratory investigations should be obtained for specific indications based on the patient history, physical examination, co morbidities and the type of surgery. A test is likely to be indicated only if it can correctly identify abnormalities and will change the diagnosis, the management plan, or the patient's outcome. Routine laboratory investigation before surgery is rampant. It seldom however changes the anaesthetic care plan or influence outcome. This research sought to find out the practice of preoperative laboratory testing at the Komfo Anokye Teaching Hospital (K. A. T. H). Methodology: This was a prospective, cross sectional study of patients undergoing elective surgery at K. A. T. H from $1^{\text {st }}$ to $31^{\text {st }}$ March 2014. A quantitative technique was used to effectively quantify laboratory results that were contained in a patient's folder before an elective surgical procedure. Close and open ended questionnaire was developed and answered by reviewing patient's folders during the pre-anaesthesia assessment. Data were analyzed using Statistical Package of Social Sciences (SSPS) version 22. Results: The average age of patients studied was 50 years. Complete blood count (CBC) is the most ordered laboratory investigation at KATH (98.8\%). Every patient had at least four (4) laboratory investigations done. At the time of preoperative assessment by the anaesthetist, laboratory investigations had already been ordered by the surgical team in almost all the patients studied (98.8\%). The anaesthetists ordered investigations in only $1.2 \%$ of the patients. When tests ordered were compared to the Canadian Anesthesiology Society (CAS) guidelines, it was found that $54.4 \%$ of renal function test ordered had no indication, as was the case with serum electrolytes $40.4 \%$, coagulation studies $39.9 \%$ and complete blood count $23.9 \%$. Conclusion: A lot of laboratory investigations requested for patients undergoing surgery at K. A. T. H have no clinical indication. Members of the surgical team are responsible for ordering the laboratory investigations. Complete blood count is the most frequently ordered investigation.
\end{abstract}

Keywords: Preoperative, Laboratory Investigations, Elective Surgery, Practice

\section{Introduction}

Preoperative tests, as a component of pre-anaesthetic assessment are traditionally considered as an important element in preoperative evaluation to determine the fitness of patients to anaesthesia and surgery [1,2]. Despite its importance, the ordering of preoperative laboratory investigation should be based on the patient's history, physical examination, co-morbidities and the risk of the surgical procedure. A test is likely to be indicated only if it can correctly identify abnormalities and will change the diagnosis, the management plan, or the patient's outcome [3, 4]. Preoperative laboratory testing should be obtained for specific clinical indications that may increase perioperative risk and not simply because the patient is to undergo a surgical procedure [5]. Routine laboratory testing before elective surgery in healthy patient have limited clinical relevance and will seldom change the anaesthetic care plan or 
influence outcome [6]. However, the use of routine laboratory investigations before elective surgery is rampant $[2]$.

In many health institutions, the practice has been that all preoperative tests and diagnostic studies are ordered by the surgeon or primary care physician. Mostly these tests are requested without specific indication based on history or physical examination but rather on speculations that the anaesthetist may "require them" to proceed with the surgery and thus avoid delays and cancellations [5, 7]. Other reasons for the ordering of preoperative laboratory investigations by surgeons include practice tradition, belief that other physicians want the tests done, medico-legal worries, concerns about surgical delays or cancellation, and lack of awareness of evidence and guidelines for ordering laboratory tests [7]. Anaesthesiologists are the perioperative medicine experts and are best qualified to establish appropriate and necessary preoperative laboratory and diagnostic tests for intra-operative anaesthesia management [5].

The concept of routine testing in all pre-surgical patients regardless of age or medical condition is no longer considered medically appropriate [5]. Selective preoperative testing other than routine testing is the gold standard for evaluating patient's fitness for anaesthesia [8]. Many societies of anaesthesia have developed standards for evaluating patients [9]. Most standards are based on factors such as ASA grading and surgical grading or type of surgery, the patient's age, exercise tolerance, medical co-morbidities and drug therapies [8-11]. The department of anaesthesia and intensive care at Komfo Anokye Teaching Hospital (KATH) recently published an institutional guideline on the practice of anaesthesia. The guideline debunked the practice of routine laboratory testing in healthy patients with no medical problem prior to an elective procedure; the absence of laboratory tests is not a reason to delay a case; Patients who have medical conditions such as kidney, liver, and thyroid disease should have appropriate laboratory tests prior to undergoing anaesthesia and surgery. Although this guideline provides indications for certain laboratory investigations, the practice has not been studied. Therefore, this research sought to find out if routine preoperative testing was practised in $\mathrm{KATH}$, who normally ordered preoperative laboratory test and the most frequently requested laboratory test for patients coming for elective surgical procedures at KATH.

\section{Methods}

This was a prospective, cross-sectional survey which involved patients undergoing elective surgical procedures at the Komfo Anokye Teaching Hospital, Kumasi, Ghana. After ethical clearance from the Committee for human research publication and ethics, a census from $1^{\text {st }}-31^{\text {st }}$ March 2014 was done to collect data from folders of patients presenting for various surgical procedures that met the inclusion criteria, and the anaesthetist who anaesthetized the patients filled the final part of the questionnaire. The study population comprised patients presenting for general surgery, gynaecological, orthopaedic, Ear, Nose and Throat, maxillofacial and ophthalmologic surgeries aged 15 years and above. Patients for emergency surgical procedures, neurological and heart surgeries as well as obstetric surgeries were excluded. The folders of all the patients presenting for elective surgery during the study period was reviewed. A quantitative technique was used to effectively quantify laboratory results that were contained in a patient's folder before an elective surgical procedure. Close and open ended questionnaire was developed and answered by reviewing patient's folders during the pre-anaesthesia assessment. Part of the questionnaire was answered by the attending anaesthetist immediately after the surgical procedure had been performed. In all a total of one hundred and sixty five patients were enrolled in the study. The data was analyzed using Statistical Package of Social Sciences SPSS version 22 and Microsoft Excel 2010. The results were presented in the form of frequency tables.

\section{Results}

All the patients studied had at least one laboratory result before anaesthetic assessment (100\%). The male to female ratio was 1:1.1. Table 1 shows the distribution of age and ASA class. It reveals that most of the study group were ASA I $(66.1 \%)$ and majority of these belonged to the $15-40$ years age group (36.4\%); only $3.0 \%$ of the ASA I patients were above 60 years of age. Overall, more patients belonged to the $40-60$ years age group (50.9\%) and all of them were either ASA I or ASA II; this same trend in ASA class was observed in all patients that were above 60 years of age $(10.3 \%)$.

Table 1. Age Distribution and ASA classification.

\begin{tabular}{lllll}
\hline \multicolumn{4}{l}{ Distribution of age (years) } & \\
\hline ASA class & $\mathbf{1 5 - 4 0}$ & $\mathbf{4 1 - 6 0}$ & above 60 & Total \\
\hline I & $60(36.4 \%)$ & $44(26.7 \%)$ & $5(3.0 \%)$ & $109(66.1 \%)$ \\
II & $1(0.6 \%)$ & $40(24.2 \%)$ & $12(7.3 \%)$ & $53(32.1 \%)$ \\
III & $3(1.8 \%)$ & $0(0.0 \%)$ & $0(0.0 \%)$ & $3(1.8 \%)$ \\
IV & $0(0.0 \%)$ & $0(0.0 \%)$ & $0(0.0 \%)$ & $0(0.0 \%)$ \\
IV & $64(38.8 \%)$ & $84(50.9 \%)$ & $17(10.3 \%)$ & $165(100 \%)$ \\
\hline
\end{tabular}

Table 2. Frequency of tests ordered.

\begin{tabular}{lll}
\hline Laboratory Test & Requested & Not requested \\
\hline Complete blood count & $163(98.8 \%)$ & $2(1.2 \%)$ \\
Renal function test & $125(75.8 \%)$ & $40(24.2 \%)$ \\
Blood grouping and matching & $105(63.6 \%)$ & $60(36.4 \%)$ \\
Serum electrolytes & $94(57.0 \%)$ & $71(43.0 \%)$ \\
Liver function test (LFT) & $48(29.1 \%)$ & $117(70.9 \%)$ \\
Urinalysis & $19(11.5 \%)$ & $146(88.5 \%)$ \\
Blood glucose & $18(10.9 \%)$ & $147(89.1 \%)$ \\
Coagulation studies & $13(7.9 \%)$ & $152(92.1 \%)$ \\
Thyroid function test (TFT) & $9(5.5 \%)$ & $156(94.5 \%)$ \\
Haemoglobin & $0(0.0 \%)$ & $165(100 \%)$ \\
Haematocrit & $0(0.0 \%)$ & $165(100 \%)$ \\
Pregnancy evaluation & $0(0.0 \%)$ & $165(100 \%)$ \\
\hline
\end{tabular}

Table 2 shows that Complete blood count (CBC) is the most frequently ordered Laboratory test for patients coming for elective surgical procedure at KATH. Out of the 165 folders reviewed, 163 representing $98.8 \%$ had a request for 
CBC, followed by Renal function test (75.8\%), Grouping and cross-matching (63.6\%), Serum electrolyte $(57.0 \%)$, Liver function test $(29.1 \%)$, Urinalysis $(11.5 \%)$, Blood Glucose $(10.9 \%)$, Coagulation studies $(7.9 \%)$, Thyroid function test (5.5\%), Haemoglobin and Haematocrit are ordered as part of another test. In none of the female patients was pregnancy test ordered. Most laboratory investigations were ordered within two weeks of surgery in $76.4 \%$ of the study population, within one month in $10.3 \%$, within 2 months in $4.2 \%$, within three months in $7.3 \%$, above three months in $0.6 \%$ and above six months in $1.2 \%$ respectively. In $81 \%$ of the study population, laboratory investigations were not repeated whereas in 19\% of cases, laboratory investigations were repeated due to abnormal values; of the $19 \%$, CBC only was the most repeated laboratory investigation representing
$15.2 \%, \mathrm{CBC}$ and Renal function test $0.6 \%, \mathrm{CBC}$, Renal function test, Serum electrolyte $1.2 \%, \mathrm{CBC}$, Renal function test, Liver function test $0.6 \%$, Random blood sugar $1.8 \%$, Renal function test only $0.6 \%$ and Thyroid function test $0.6 \%$. Furthermore, it was observed that same tests were repeated two times in $18.8 \%$ of the study population, three times in $1.2 \%$ and four times in $0.6 \%$.

Table 3 shows the distribution of laboratory investigations according to Age, ASA classification and category of surgery. It should be noted that of the ASA I patients undergoing minor surgery, tests such as $\mathrm{CBC}$, Coagulation studies, serum electrolyte, Renal function test and Liver function test were done in $22 \%, 38 \%, 10 \%, 14 \%$ and $6 \%$ of the study population respectively. This trend was also seen with the ASA II patients.

Table 3. Distribution of laboratory investigations according to Age, ASA classification and category of surgery.

\begin{tabular}{|c|c|c|c|c|c|c|c|}
\hline & \multicolumn{7}{|l|}{ ASA I } \\
\hline & \multicolumn{7}{|c|}{ CATEGORY OF SURGERY } \\
\hline & \multicolumn{3}{|c|}{ Major Procedure } & \multirow{3}{*}{$\%$ Study Population } & \multicolumn{3}{|c|}{ Minor Procedure } \\
\hline & \multicolumn{3}{|c|}{ AGE RANGE } & & \multicolumn{3}{|c|}{ AGE RANGE } \\
\hline & $15-40$ years & 41-60YRS & Above 60YRS & & $15-40$ years & 41-60YRS & Above 60YRS \\
\hline CBC & 36 & 32 & 3 & $43 \%$ & 22 & 12 & 2 \\
\hline COA STUD & 0 & 0 & 0 & $0 \%$ & 4 & 1 & 0 \\
\hline SE & 28 & 24 & 1 & $56 \%$ & 4 & 4 & 1 \\
\hline RFT & 25 & 29 & 1 & $44 \%$ & 8 & 7 & 2 \\
\hline LFT & 14 & 8 & 1 & $48 \%$ & 2 & 0 & 1 \\
\hline
\end{tabular}

Table 3. Continue.

\begin{tabular}{|c|c|c|c|c|c|c|c|}
\hline & \multicolumn{7}{|l|}{ ASA II } \\
\hline & \multicolumn{7}{|c|}{ CATEGORY OF SURGERY } \\
\hline & \multicolumn{3}{|c|}{ Major Procedure } & \multirow{3}{*}{ \% Study Population } & \multicolumn{3}{|c|}{ Minor Procedure } \\
\hline & \multicolumn{3}{|l|}{ AGE RANGE } & & \multicolumn{3}{|l|}{ AGE RANGE } \\
\hline & $15-40$ years & 41-60YRS & Above 60YRS & & $15-40$ years & 41-60YRS & Above 60YRS \\
\hline $\mathrm{CBC}$ & 1 & 36 & 7 & $27 \%$ & 0 & 4 & 5 \\
\hline COA STUD & 0 & 7 & 0 & $54 \%$ & 0 & 1 & 0 \\
\hline SE & 1 & 21 & 2 & $26 \%$ & 0 & 0 & 5 \\
\hline LFT & 0 & 9 & 5 & $29 \%$ & 0 & 1 & 4 \\
\hline
\end{tabular}

Table 3. Continue.

\begin{tabular}{|c|c|c|c|c|c|c|c|c|}
\hline & \multicolumn{7}{|l|}{ ASA III } & \multirow{5}{*}{$\begin{array}{l}\text { \% Study } \\
\text { Population }\end{array}$} \\
\hline & \multicolumn{7}{|c|}{ CATEGORY OF SURGERY } & \\
\hline & \multirow{2}{*}{\multicolumn{3}{|c|}{$\begin{array}{l}\text { Major Procedure } \\
\text { AGE RANGE }\end{array}$}} & \multirow{3}{*}{$\begin{array}{l}\text { \% Study } \\
\text { Population }\end{array}$} & \multicolumn{3}{|c|}{ Minor Procedure } & \\
\hline & & & & & AGE RANGE & & & \\
\hline & $15-40$ years & 41-60YRS & Above 60YRS & & $15-40$ years & 41-60YRS & Above 60YRS & \\
\hline $\mathrm{CBC}$ & 3 & 0 & 0 & $2 \%$ & 0 & 0 & 0 & $0 \%$ \\
\hline COA STUD & 0 & 0 & 0 & $0 \%$ & 0 & 0 & 0 & $0 \%$ \\
\hline SE & 3 & 0 & 0 & $3 \%$ & 0 & 0 & 0 & $0 \%$ \\
\hline RFT & 3 & 0 & 0 & $2 \%$ & 0 & 0 & 0 & $0 \%$ \\
\hline LFT & 3 & 0 & 0 & $6 \%$ & 0 & 0 & 0 & $0 \%$ \\
\hline
\end{tabular}

Table 4 reveals that at the time of preoperative assessment by the anaesthetist, laboratory investigations have already been ordered by the surgical team in almost all the patients studied $98.8 \%$. The anaesthetist ordered for investigations in only $1.2 \%$ of the patients. The table also shows that of the test ordered by the surgeons, the anaesthetist found no indication for the tests in $20.6 \%$ of the patients, but agreed that in $79.4 \%$ of the cases, the tests were indicated. This assessment was based on the ASA classification and type of surgery.
Table 4. Who ordered laboratory investigations and number of patients who had tests that were indicated or not indicated at Anaesthetist's preoperative review?

\begin{tabular}{lll}
\hline Who orders lab investigations? & Number & Percentage \\
\hline Surgical team & 163 & 98.8 \\
Anaesthetist & 2 & 1.2 \\
Total & 165 & 100 \\
Test indicated & 131 & 79.4 \\
Test not indicated & 34 & 20.6 \\
Total & 165 & 100 \\
\hline
\end{tabular}


When the tests done were assessed by the CAS guidelines (tables 5 and 6), it was found that of the 124 (76.1\%) patients in which $\mathrm{CBC}$ was indicated, abnormal results were found in only $39(31.5 \%)$ of them and of the $39(23.9 \%)$ in which it was not indicated, $2(5.1 \%)$ had abnormal result. For coagulation studies, out of the $8(61.5 \%)$ who had indication, $6(75 \%)$ had abnormal result and in the $5(39.5 \%)$ in whom the test was not indicated, none $(0 \%)$ had an abnormal result. Tests were indicated in $56(59.6 \%)$ for Serum electrolyte, yet no abnormality $(0 \%)$ was found in the results of all of them. Abnormal result was found in $3(7.9 \%)$ of the $38(40.4 \%)$ patients in whom serum electrolytes was not indicated. Renal function test was indicated in $57(45.6 \%)$ of the study population and $12(21.1 \%)$ had abnormal results. Of the 68 $(54.4 \%)$, in whom the test was not indicated, abnormal result was found in $5(7.4 \%)$.

Table 5. The Canadian Anaesthesiology Society Guidelines for preoperative laboratory testing.

\begin{tabular}{|c|c|}
\hline Test & indications \\
\hline Complete blood count & $\begin{array}{l}\text { - Patient more than } 60 \text { year of age } \\
\text { - Major surgery requiring "group and screen" } \\
\text { or "group and match" } \\
\text { - Chronic cardiovascular, pulmonary, renal or } \\
\text { hepatic disease } \\
\text { - Malignancy } \\
\text { - Known or suspected anaemia, bleeding } \\
\text { diathesis or myelo-suppression } \\
\text { - Anticoagulant therapy }\end{array}$ \\
\hline Coagulation profile & $\begin{array}{l}\text { - Anticoagulant therapy } \\
\text { - Bleeding diathesis, family history of bleeding } \\
\text { disorder } \\
\text { - History of DVT or pulmonary embolism } \\
\text { - Liver disease, renal failure } \\
\text { - Malignancy with concurrent radio- } \\
\text { chemotherapy }\end{array}$ \\
\hline $\begin{array}{l}\text { Electrolyte and } \\
\text { creatinine levels }\end{array}$ & $\begin{array}{l}\text { - Age more than } 60 \text { years } \\
\text { - Hypertension } \\
\text { - Renal disease } \\
\text { - Diabetes } \\
\text { - Pituitary or adrenal disease } \\
\text { - Digoxin or diuretic therapy, or other drug } \\
\text { therapies affecting electrolytes }\end{array}$ \\
\hline
\end{tabular}

Table 6. Number of investigations that were indicated or not indicated according to the Canadian Anesthesiology Society guidelines for preoperative laboratory testing.

\begin{tabular}{lll}
\hline Laboratory test & Indicated & Not indicated \\
\hline Complete blood count (\%) & $124 / 163(76.1)$ & $39 / 163(23.9)$ \\
Serum electrolytes (\%) & $56 / 94(59.6)$ & $30 / 94(40.4)$ \\
Renal function test (\%) & $57 / 125(46.6)$ & $68 / 125(54.6)$ \\
Coagulation studies (\%) & $8 / 13(61.5)$ & $5 / 13(39.5)$ \\
\hline
\end{tabular}

\section{Discussions}

Our study shows that routine preoperative testing is practised in Komfo Anokye Teaching Hospital, considering the number of patients who had tests that were not indicated according to the Canadian Anaesthesiologist Society guidelines and the assessment of the reviewing Anaesthetists (20.6\%). Furthermore, abnormal results were few in most of the patients in whom the tests were indicated. Our finding agrees with that of Kaplan et al [12]. In their study, a retrospective review of charts of over 2000 elective surgical patients who underwent battery of tests including complete blood cell count, differential blood count, prothrombin time, glucose level, serum electrolytes, creatinine, platelet count, etc, demonstrated that only $96 \quad(22 \%)$ tests revealed abnormalities; of the 96 abnormal test results, only 10 could not be determined by history and examination, and of the 10 , only 4 were of actual clinical significance. In the early 1940 when preoperative assessment evolved, preoperative investigations were based on thorough history and physical examination during preoperative visit. Laboratory test ordered were done selectively to confirm diagnosis [9]. However in the late 1960 when test ordering became easier because of the introduction of biochemical auto-analyzers routine testing became common without recourse to the economic burden on patients. The idea of routine testing was equated to efficient care [2]. Many hospitals adopted this behaviour to perform series of laboratory test prior to surgical operations with the assumption that voluminous information would enhance the safety of surgical patients [2]. The practice continued without any scientific evidence until Kaplan et al (1985) published an academic paper to debunk the practice. During the past three decades, the practice of routine investigations prior to surgery has been challenged by several academic publications as it involves a sizable cost without significant benefit to millions of patients undergoing surgery $[2,8,11,13]$.

Our study also reveals that, it is the surgical team and their members that order preoperative laboratory investigations at KATH (98.8\%). The laboratory investigations are ordered before patients visit the anaesthetist at the pre-anaesthetic clinic. This finding is supported by Patey et al who showed that in various hospitals across the province of Ontario, Anesthesiologists are often not the professionals responsible for ordering pre-operative tests [14]. Our study also shows that anaesthetist were comfortable with the test ordered by surgeons in $79.4 \%$ of the patients. However, the observation that $38 \%$ of the ASA I patients, who had minor surgeries, had serum electrolytes tests, puts to question the judgement of the anaesthetist who probably may have also seen some of the tests as routine investigations for all surgical patients. This is in contrast to another study in which preoperative laboratory tests ordered by the surgeon were compared to those tests considered indicated by one of several anesthesiologists for ASA physical status I and II versus ASA physical status III patients; an average of $72.5 \%$ of tests ordered by surgeons was considered not indicated by the anesthesiologists; ASA physical status III patients had significantly fewer unindicated laboratory orders than did ASA physical status I and II patients [8]. Furthermore, a study by Vogt et al found that large percentages of preoperative tests ordered by surgeons were not indicated and that eliminating un-indicated tests, would cut hospital revenues in a climate where testing is fee-for-service and would save the hospital money in a managed-care or capitated system [15]. 
Ordering of preoperative investigations can be done by both the surgeons and the anaesthetist during the preparative phase for a patient going for an elective surgery. Both anaesthesiologists and surgeons are equally guilty when it comes to the ordering of "routine testing". Anaesthesiologists often, but not inevitably, will check the results of the tests that they order; however it is the experience of many that surgeons rarely check the results or respond to abnormal results of the tests that they order [16].

Surgeons will state that they order the tests because "anaesthesia" wants them to do so, and some anaesthesiologists say that they order tests to protect themselves legally. Neither explanation is scientifically rigorous, nor do they demonstrably improve care [17].

Preoperative testing should be individualized for each patient. The preoperative history and physical examination should be the determinants of any testing applicable to each particular patient. Indeed, the ordering of non-demonstrablynecessary tests as a routine is not appropriate [16].

An interview study using semi-structured interviews with anaesthesiologists and surgeons by Patey et al [14] stated that; both groups felt that they did not need to order or review a CXR or ECG to adequately do their job when performing a low-risk surgical procedure on a healthy patient, they made conflicting comments as to who exactly was responsible for ordering the pre-operative tests and responses within each professional group varied. For example, several anaesthesiologists stated that they should have complete autonomy as to what tests should be ordered whereas others noted that within their hospital it was not their responsibility to order the pre-operative tests. Conversely, some surgeons noted that pre-operative test ordering was the responsibility of the anaesthesiologists, while others mentioned that they were the most responsible physician in the operating room and as such had the ultimate responsibility to understand the whole picture. They further stated that both anaesthesiologists and surgeons identified factors within their environment that affected their decision to order preoperative tests. There was considerable disagreement as to whether time constraint was a factor in test ordering practice. There were also reports of a gap between their motivation and practice. Both anaesthesiologists and surgeons mentioned that if hospitals made sure that all pre-operative testing was conducted by only anaesthesiologists and took the ordering out of the hands of the surgeons, unnecessary routine testing could be reduced [17].

The lack of clarity about who is responsible for routine test ordering appears to lead to a propensity to order tests just in case' they are expected by another colleague. A surgeon may order the tests 'in case' the attending anaesthesiologist needs it and in hopes that the patient will move smoothly through the pre-admission assessment process. The anaesthesiologist who sees the patient prior to the surgery orders the tests in case' the attending anaesthesiologist needs them and could not cancel tests ordered by the surgeon because they have not identified the reason for ordering the tests. Furthermore, the anaesthesiologists interviewed reported they seldom reviewed test results when caring for low-risk patients in the operating room. The interesting thing about the team influence is that although anaesthesiologists and surgeons greatly influence whether pre-operative test are ordered by another team member, these clinicians rarely have direct contact with one another and communication is difficult [17]. A study at the National hospital Sri Lanka, found House Officers to be the worse culprits when it comes to ordering non - indicated investigations followed by medical officers (anaesthesia) and anaesthesia registrars. Senior residents, anaesthetist and consultants showed adherence to guidelines [18]. Where standards exist it may not matter who orders preoperative investigations provided it is based on the patient's history, physical examination, category of the surgery, and drug therapy.

Our study also shows that $\mathrm{CBC}$ is the most ordered laboratory investigation at KATH (98.8\% of respondents), followed by RFT (75.8\%), G\&M (63.6\%), SE (57.0\%), LTF (29.1\%), Urinalysis (11.5\%), Blood Glucose (10.9\%) Coagulation studies $(7.9 \%)$, TFT (5.5\%), Haemoglobin and Haematocrit were not ordered alone but as part of $\mathrm{CBC}$; in most cases of $\mathrm{CBC}$ ordered, the anaesthetist only needed haemoglobin/ haematocrit levels. Pregnancy test was omitted in all the female patients. Pregnancy has an important bearing in the perioperative care of a woman. The American Society of Anesthesiologists recommends pregnancy testing for all women of child bearing age. History may not be completely reliable to exclude pregnancy [19]. Every patient had at least four (4) laboratory investigations done regardless of the type of surgical procedure, CBC 98.8\% RFT 75.8\% G\&M 63.6\% and SE $57.0 \%$. In $76.4 \%$ of patients studied, investigations done were within two weeks and $21.8 \%$ within six months to surgery. CBC tests within three months of request were only repeated, if there was an abnormality in haemoglobin level; this result support literature that, normal laboratory results obtained within 4 months of surgery should not be repeated [20].

\section{Conclusion}

Routine laboratory testing is practised in Komfo Anokye Teaching Hospital. Members of the surgical team are responsible for ordering the laboratory investigations. Complete blood count is the most frequently ordered investigation and results within four months of surgery that are not abnormal, are accepted for surgery. Laboratory investigations are mostly ordered within 2 weeks of surgery. It is recommended that a more comprehensive guideline be developed to help in the ordering of preoperative laboratory investigation. Ordering of preoperative investigation should be the responsibility of the anaesthetist during preoperative assessment; the practice of surgeons ordering preoperative laboratory investigations for the anaesthetist should be minimised and where necessary haemoglobin estimation should be ordered instead of a complete blood count to reduce the cost to the patient and the insurance schemes. 


\section{Statement on Conflict of Interest}

The authors declare that there is no conflict of interest regarding the publication of this paper.

\section{References}

[1] Standards and Practice Parameters, ASA House of Delegates. Routine Preoperative Laboratory and Diagnostic Screening - Google Search [Internet]. 2008 [cited 2013 Oct 14].

[2] Kumar A, Srivastava U. Role of routine laboratory investigations in preoperative evaluation. J Anaesthesiol Clin Pharmacol. 2011; 27: 174-9.

[3] Hepner DL. The role of testing in the preoperative evaluation. Cleve Clin J Med. 2009; 76: 22-7.

[4] Feely MA, Collins CS, Daniels PR, Kebede EB, Jatoi A, Mauck KF. Preoperative testing before noncardiac surgery: guidelines and recommendations. Am Fam Physician. 2013; 87: 414-8.

[5] Fischer SP, Bader AM, Sweitzer B. Preoperative Evaluation. In: Ronald D Miller, editor. Millers Anesthesia $7^{\text {th }}$ edition. Elsevier Churchill Livingstone 2009: chapter-34.

[6] Smetana GW, Macpherson DS. The case against routine preoperative laboratory testing. Med Clin North Am. 2003; 87: 7-40.

[7] Brown SR, Brown J. Why do physicians order unnecessary preoperative tests? Fam Med. 2011; 43: 338-43.

[8] Apfelbaum JL, Connis RT, Nickinovich DG, Pasternak LR, Arens JF et al. Practice advisory for preanesthesia evaluation: an updated report by the American Society of Anesthesiologists Task Force on Preanesthesia Evaluation. Anesthesiology. 2012; 116: 522-38.

[9] National Collaborating Centre for Acute Care. NICE Guideline for Preoperative Investigations in Patients Undergoing Elective Surgery 2003 [cited 2013 Oct 10].
[10] Routine Preoperative Lab Tests for Adult patients (age $\geq 16$ years) undergoing Elective Surgery - GRIDFINAL Dec.10.pdf [Internet]. 2010 [cited 2014 Feb 17].

[11] Merchant R, Chartrand D, Dain S, Dobson G, Kurrek MM et al. Guidelines to the practice of anesthesia. Can J Anesth/ J Can Anesth 2014: 61: 46-71.

[12] Kaplan EB, Sheiner LB, Boeckmann AJ, Roizen MF, Beal SL et al. The usefulness of preoperative laboratory screening. JAMA. 1985; 253: 3576-81.

[13] Johnson H, Knee-Loli S, Butler TA, Munoz E, Wise L. Are routine preoperative laboratory screening tests necessary to evaluate ambulatory surgical patients? Surgery. 1988; 104: $639-45$.

[14] Chung F, Yuan H, Yin L, Vairavanathan S, Wong DT. Elimination of Preoperative Testing in Ambulatory Surgery. Anesth Analg. 2009; 108: 467-75.

[15] Vogt AW, Henson LC. Unindicated preoperative testing: ASA physical status and financial implications. J Clin Anesth. 1997; 9: 437-41.

[16] Patey AM, Islam R, Francis JJ, Bryson GL, Grimshaw JM. Anesthesiologists' and surgeons' perceptions about routine pre-operative testing in low-risk patients: application of the Theoretical Domains Framework (TDF) to identify factors that influence physicians' decisions to order pre-operative tests. Implement Sci. 2012; 7: 52.

[17] Thelma Z Korpman. Routine Preoperative Laboratory and Diagnostic Screening. 2014.

[18] Ranasinghe P, Perera YS, Abayadeera A. Preoperative Investigations in Elective Surgery: Practices and Costs at the National Hospital of Sri Lanka. Sri Lankan Journal of Anaesthesiology 2014; 18: 29.

[19] Practice advisory for preanesthesia evaluation: a report by the American Society of Anesthesiologists Task Force on Preanesthesia Evaluation. Anesthesiology. 2002; 96: 485-96.

[20] Macpherson DS, Snow R, Lofgren RP. Preoperative screening: value of previous tests. Ann Intern Med 1990; 113: 969-73. 